\title{
Editorial: Primary Glial and Immune Cell Pathology in Neurodegenerative Diseases
}

\author{
András Lakatos ${ }^{1,2 *}$ and Gabor Petzold ${ }^{3,4 *}$ \\ 1 John van Geest Centre for Brain Repair, Department of Clinical Neurosciences, University of Cambridge, Cambridge, \\ United Kingdom, ${ }^{2}$ Wellcome Trust-MRC Cambridge Stem Cell Institute, Cambridge, United Kingdom, ${ }^{3}$ German Center for \\ Neurodegenerative Diseases (DZNE), Bonn, Germany, ${ }^{4}$ Division of Vascular Neurology, University Hospital Bonn, Bonn, \\ Germany
}

Keywords: glia, astrocyte, microglia, immune cell, neurodegeneration, Alzheimer's disease, Parkinson's disease, glaucoma

\section{Editorial on the Research Topic}

Primary Glial and Immune Cell Pathology in Neurodegenerative Diseases

Non-neuronal cells in the brain have been proposed as key modulators of neuronal network function in health and in neurological disease. Until recently, the involvement of glial cellsincluding astrocytes, microglial cells, oligodendrocytes and their progenitors-and infiltrating immune cells in neurodegenerative diseases has been merely viewed as a secondary adaptive response to disease-specific neuronal pathology. It emerges that glial and other non-neuronal cells

\section{OPEN ACCESS}

Edited and reviewed by: Bruce Miller,

University of California, San Francisco, United States

*Correspondence: András Lakatos AL291@cam.ac.uk Gabor Petzold

gabor.petzold@dzne.de

Specialty section:

This article was submitted to Dementia and Neurodegenerative

Diseases,

a section of the journal

Frontiers in Neurology

Received: 27 August 2021 Accepted: 21 September 2021

Published: 18 October 2021

Citation:

Lakatos A and Petzold G (2021) Editorial: Primary Glial and Immune Cell Pathology in Neurodegenerative Diseases. Front. Neurol. 12:765376. doi: 10.3389/fneur.2021.765376 can also be directly affected by neurodegenerative cues, worsening neuronal dysfunction $(1,2)$. This has highlighted new potential avenues in targeting disease pathways in a broad spectrum of neurodegenerative conditions, which would require teasing apart primary pathology from secondary processes in multiple cell types.

In this issue we brought together some of the leaders in glial cell biology and neurodegenerative disease research to cover recent advances in pathomechanistic studies informing novel experimental treatment strategies in six review articles.

The Research Topic starts with two articles discussing the emerging role of astrocytes in neurodegeneration. One of the most argued, and therapeutically relevant issues, is the extent to which morphological, gene expression and signaling changes characterize a primary cellautonomous astrocyte pathology or a secondary adaptive or "reactive" response to damage. There is a consensus in the field to steer away from the less reliable phenotypic classifications and rather assess the function of various astrocytic states in individual disease models to help reveal potential treatment targets (3). What complicates this assessment is the growing evidence that astrocytes already represent a large and heterogenous cell population $(4,5)$. Monterey et al. review the "many faces" of astrocytes, which can represent both a regional cell diversity and a broad functional spectrum in disease with multiple targetable elements. They discuss recent advances in sequencing technologies, and how they can be used to distinguish pathological cell states and signaling disturbances in Alzheimer's disease (AD). The article by García-Bermúdez et al. emphasizes that glaucoma, a common eye disease that damages the optic nerve and the retina, shares glia-mediated mechanisms with many neurodegenerative diseases, such as $\mathrm{AD}$. The concept of common pathological processes between the eye and the brain is also supported by observations of retinal changes in $\mathrm{AD}$ patients, forming the basis of an emerging ophthalmological diagnostic opportunity for neurodegenerative disorders (6). Furthermore, the authors provide a broad overview on retinal glia-ganglion cell interactions as potential therapeutic targets. Altogether, 
these articles highlight the importance of distinguishing homoeostatic and detrimental astrocyte responses for identifying potentially targetable pathological signaling cascades.

Another major advance concerns microglial transcriptomic and inflammatory signaling changes in neurodegenerative pathologies. Similar to astrocytes, microglia may adapt to neuronal injuries by regaining their homeostatic function but can rapidly escalate the expression of inflammatory mediators as part of the innate immune response. How the adaptive immune response and other risk factors trigger this process in neurodegeneration have been an exciting topic in research over the last decade $(7,8)$. A minireview by Candlish and Hefendehl highlight the recent significant advances in this field. In particular, they overview mechanisms that govern the transition of microglia into various subtypes during the neurodegenerative process. A particularly interesting angle is the discussion about the risks that lifestyle factors and aging processes impose on cell phenotype changes. Nitsch et al. then provides a detailed overview on the contribution of microglial signaling to $\mathrm{AD}$ pathology with a specific focus on interleukin-23 (IL-23). This paper sheds light on mechanisms by which $\mathrm{p} 40$, an IL-23 subunit can be released by microglial cells upon exposure to amyloid-beta $(\mathrm{A} \beta)$, a central molecule in $\mathrm{AD}$ pathogenesis. One of the key messages of this review is that $\mathrm{p} 40$ appears to establish a new link between $\mathrm{A} \beta$ and neuroinflammation, possibly via Th17 cells, astrocytes and microglia. Although the identity of effector cells and pathways induced by IL-23 require further elucidation, blocking or neutralizing antibodies for IL-23 may provide promises in reducing cerebral amyloid load or soluble $A \beta$ species (9), which may attract therapeutic interests. Since antiinflammatory or current antibody treatment approaches have yet to improve the clinical outcome in neurodegeneration, for instance in $\operatorname{AD}$ patients $(10,11)$, the articles in this special issue well serve the purpose of highlighting potential target options for more effective strategies.

The final two articles wrap up the recently revealed aspects of interactions between astrocytes, microglia, peripheral immune cells, and their effect on neuronal networks. Pietrowski et al. reviews the growing evidence of purinergic signaling and its breakdown between glial cells and neurons. This is of particular relevance to non-cell autonomous pathomechanisms in neurodegeneration $(1,7)$, which can worsen neuronal network function, leading to cognitive or motor decline. This paper also brings up the issue of emerging major transcriptional and functional differences between human and mouse astrocytes and microglia $(12,13)$, including expression of genes that are a pre-requisite for their interactions with neurons. Considering these potential differences is crucial when interpreting results in mouse disease models that do not entirely recapitulate the human

\section{REFERENCES}

1. Taylor JP, Brown RH, Cleveland DW. Decoding ALS: from genes to mechanism. Nature. (2016) 539:197-206. doi: 10.1038/nature20413

2. De Strooper B, Karran E. The cellular phase of Alzheimer's disease. Cell. (2016) 164:603-15. doi: 10.1016/j.cell.2015.12.056 pathobiological phenotype (14). Another timely issue related to glial cell communication concerns immune cells, a topic which has emerged onto the central stage of neurodegeneration research. Copas et al. put this into an interesting perspective. They describe how the genetic risk in Parkinson's Disease (PD) may affect glial cells and conspire with peripheral infections during lifetime, predisposing to a chronic neuroinflammatory response. The authors follow us through the ways infiltrating $\mathrm{T}$ cells could play a central role, triggered by antigen-presenting microglia. They argue that this may also lead to altered astrocytic inflammatory responses, and consequently contribute to the loss of dopaminergic neurons. The broad overview of the above disease-related pathways illuminates the role of infection and peripheral immune activation as important risk factors in neurodegenerative diseases.

\section{CONCLUSIONS}

Overall, the review articles in this issue remind us of the multiple cell-types that are primarily involved in disease, and also focus on those cell populations that are not innate in the brain. The discussions highlight the need for systems biology approaches to distinguish initiating molecular disturbances that can be obscured by secondary homeostatic responses in many cell populations. Recent examples have already shown us how new technologies and platforms, such as single cell or spatial transcriptomics and human stem cell-based or brain organoids could resolve the above problem (1517). We anticipate that the emerging data demonstrating human-specific differences in pathogenesis will transform translational science and personalized treatment strategies in this decade.

\section{AUTHOR CONTRIBUTIONS}

All authors listed have made a substantial, direct and intellectual contribution to the work, and approved it for publication.

\section{ACKNOWLEDGMENTS}

The Guest Editors would like to express their gratitude to all authors of this special issue and to reviewers Aviva Tolkovsky, Claire S. Durrant, Lies De Groef, Ed Levine, Alexei Verkhratsky, Aude Panatier, Sabina Tahirovic, Karen Gertz, Carole Escartin, Alberto Serrano-Pozo, Michela Deleidi, and Ayse Ulusoy. AL had been supported by grants from the Medical Research Council UK (MR/P008658/1), the Wellcome Trust (204845/Z/16/Z) and the Evelyn Trust. 
5. Ben Haim L, Rowitch DH. Functional diversity of astrocytes in neural circuit regulation. Nat Rev Neurosci. (2016) 18:31-41. doi: 10.1038/nrn.2016.159

6. Hadoux X, Hui F, Lim JK, Masters CL, Pébay A, Chevalier S, et al. Non-invasive in vivo hyperspectral imaging of the retina for potential biomarker use in Alzheimer's disease. Nat Commun. (2019) 10:1-12. doi: 10.1038/s41467-019-12242-1

7. Heneka MT, Kummer MP, Latz E. Innate immune activation in neurodegenerative disease. Nat Rev Immunol. (2014) 14:46377. doi: 10.1038/nri3705

8. Herz J, Filiano AJ, Smith A, Yogev N, Kipnis J. Myeloid cells in the central nervous system. Immunity. (2017) 46:94356. doi: 10.1016/j.immuni.2017.06.007

9. Vom Berg J, Prokop S, Miller KR, Obst J, Kälin RE, LopateguiCabezas I, et al. Inhibition of IL-12/IL-23 signaling reduces Alzheimer's diseasea-like pathology and cognitive decline. Nat Med. (2012) 18:18129. doi: $10.1038 / \mathrm{nm} .2965$

10. Gyengesi E, Münch G. In search of an anti-inflammatory drug for Alzheimer disease. Nat Rev Neurol. (2020) 16:131-2. doi: 10.1038/s41582-019-0307-9

11. Mullard A. Controversial Alzheimer's drug approval could affect other diseases. Nature. (2021) 595:162-3. doi: 10.1038/d41586-021-01763-9

12. Han $X$, Chen $M$, Wang F, Windrem $M$, Wang S, Shanz S, et al. Forebrain engraftment by human glial progenitor cells enhances synaptic plasticity and learning in adult mice. Cell Stem Cell. (2013) 12:34253. doi: 10.1016/j.stem.2012.12.015

13. Zhou Y, Song WM, Andhey PS, Swain A, Levy T, Miller KR, et al. Human and mouse single-nucleus transcriptomics reveal TREM2dependent and TREM2-independent cellular responses in Alzheimer's disease. Nat Med. (2020) 26:131-42. doi: 10.1038/s41591-019-0 695-9

14. Mordes DA, Morrison BM, Ament XH, Cantrell C, Mok J, Eggan P, et al. Absence of survival and motor deficits in 500 repeat C9ORF72
BAC mice. Neuron. (2020) 108:1-9. doi: 10.1016/j.neuron.2020.0 8.009

15. Camp JG, Badsha F, Florio M, Kanton S, Gerber T, Wilsch-Bräuninger $\mathrm{M}$, et al. Human cerebral organoids recapitulate gene expression programs of fetal neocortex development. Proc Natl Acad Sci. (2015) 112:201520760. doi: 10.1073/pnas.1520760112

16. Giandomenico SL, Mierau SB, Gibbons GM, Wenger LM, Masullo L, Sit $\mathrm{T}$, et al. Cerebral organoids at the air-liquid interface generate diverse nerve tracts with functional output. Nat Neurosci. (2019) 22:66979. doi: 10.1038/s41593-019-0350-2

17. Mansour AA, Gonçalves JT, Bloyd CW, Li H, Fernandes S, Quang D, et al. An in vivo model of functional and vascularized human brain organoids. Nat Biotechnol. (2018) 36:432-41. doi: 10.1038/nbt.4127

Conflict of Interest: The authors declare that the research was conducted in the absence of any commercial or financial relationships that could be construed as a potential conflict of interest.

Publisher's Note: All claims expressed in this article are solely those of the authors and do not necessarily represent those of their affiliated organizations, or those of the publisher, the editors and the reviewers. Any product that may be evaluated in this article, or claim that may be made by its manufacturer, is not guaranteed or endorsed by the publisher.

Copyright (c) 2021 Lakatos and Petzold. This is an open-access article distributed under the terms of the Creative Commons Attribution License (CC BY). The use, distribution or reproduction in other forums is permitted, provided the original author(s) and the copyright owner(s) are credited and that the original publication in this journal is cited, in accordance with accepted academic practice. No use, distribution or reproduction is permitted which does not comply with these terms. 